\title{
Post-stroke seizures are clinically underestimated
}

\author{
Carla Bentes ${ }^{1,2}$ (D) Hugo Martins ${ }^{3} \cdot$ Ana Rita Peralta $^{1,2} \cdot$ Carlos Casimiro $^{4} \cdot$ Carlos Morgado $^{2,4} \cdot$ \\ Ana Catarina Franco $^{1} \cdot$ Ana Catarina Fonseca $^{1,2} \cdot$ Ruth Geraldes $^{1,2} \cdot$ Patrícia Canhão $^{1,2} \cdot$ Teresa Pinho e Melo $^{1,2}$. \\ Teresa Paiva $^{5} \cdot$ José M. Ferro $^{1,2}$
}

Received: 15 June 2017 / Revised: 29 July 2017 / Accepted: 31 July 2017 / Published online: 14 August 2017

(C) Springer-Verlag GmbH Germany 2017

\begin{abstract}
Cerebrovascular disease is the leading cause of epilepsy in adults, although post-stroke seizures reported frequency is variable and few studies used EEG in their identification. To describe and compare EEG and clinical epileptic manifestations frequency in patients with an anterior circulation ischaemic stroke. Prospective study of acute anterior circulation ischaemic stroke patients, consecutively admitted to a Stroke Unit over 24 months and followed-up for 1 year. All patients underwent standardized clinical and diagnostic assessment. Seizure occurrence was clinically evaluated during hospitalization and by a telephone interview at 6 months and a clinical appointment at 12 months after stroke. Video-EEG was performed in the first $72 \mathrm{~h}$ (1st EEG), daily after the 1st EEG for the first 7 days after the stroke, or later if neurological worsening, at discharge, and at 12 months. 151 patients were included (112 men) with a mean age of 67.4 (11.9) years. In the 1st year after
\end{abstract}

Electronic supplementary material The online version of this article (doi:10.1007/s00415-017-8586-9) contains supplementary material, which is available to authorized users.

Carla Bentes

ccbentes@gmail.com

$1 \quad$ EEG/Sleep Laboratory/Stroke Unit, Department of Neurosciences and Mental Health (Neurology), Hospital de Santa Maria, CHLN, Avenida Professor Egas Moniz, 1649-035 Lisbon, Portugal

2 Faculty of Medicine, University of Lisbon, Lisbon, Portugal

3 Department of Medicine 1.2., Hospital de São José, CHLC, Lisbon, Portugal

4 Department of Neuroradiology, Hospital de Santa Maria, CHLN, Lisbon, Portugal

5 Eletroencefalography and Clinic Neurophysiology Centre (CENC), Lisbon, Portugal stroke, 38 patients (25.2\%) had an epileptic seizure. During hospitalization, 27 patients (17.9\%) had epileptiform activity (interictal or ictal) in the EEG, 7 (25.9\%) of them electrographic seizures. During the first week after stroke, 22 (14.6\%) patients had a seizure and 4 (2.6\%) non-convulsive status epilepticus criteria. Five $(22.7 \%)$ acute symptomatic seizures were exclusively electrographic. At least one remote symptomatic seizure occurred in $23(16 \%)$ patients. In the first 7 days after stroke, more than one-fifth of patients with seizures had exclusively electrographic seizures. Without a systematic neurophysiological evaluation the frequency of post-stroke seizures are clinically underestimated.

Keywords Ischaemic stroke - Symptomatic seizures · Epilepsy $\cdot$ EEG $\cdot$ Interictal epileptiform activity $\cdot$ Electrographic seizures

\section{Introduction}

Post-stroke seizures identification has many implications for clinical practice. One the one hand, epilepsy diagnosis can be made to a patient with at least one remote symptomatic seizure after stroke [1] since it has a recurrence probability of $71.5 \%$ [2]. One the other hand, although there is no quality evidence for the recommendation of acute symptomatic post-stroke seizures secondary prevention [3], this seems to be common in many centres in the acute stroke setting, to prevent additional metabolic burden [4].

However, the reported frequency of seizures after an ischemic stroke is variable (2-67\%) [5] possibly due to different study methodologies. One limitation of this frequency analysis has been the lack (in the vast majority of studies) of an electroencephalographic record [6]. In a retrospective study of acute brain injury patients submitted to 
EEG monitoring, most seizures (92\%) were electroencephalographic without apparent clinical manifestations [7]. More specifically, $9 \%$ of patients with an acute ischemic lesion had non-convulsive seizures and $7 \%$ had criteria for non-convulsive status epilepticus [7]. Thus, in the absence of an EEG record, the frequency of post-stroke seizures may be underestimated. Moreover, the frequency of electrographic seizures in patients with ischemic stroke who do not require admission to an intensive care unit and EEG monitoring is unknown, although epileptic seizures [2, 8] and ictal/interictal EEG discharges have been associated with stroke unfavourable outcome [9-11].

This paper aims to prospectively describe and compare the frequency of EEG and clinical epileptic manifestations in patients with an anterior circulation ischaemic stroke admitted to a Stroke Unit.

\section{Methods}

Prospective study of consecutive patients with an acute anterior circulation ischaemic stroke, admitted to the Stroke Unit of a Neurology Department over a period of 24 months (between October 2011 and October 2013) and followed-up for 12 months (October 2012 to October 2014). The study was approved by the Ethics Committee of Centro Hospitalar Lisboa Norte ("Comissão de Ética para a Saúde").

The following inclusion criteria were used:

1. Acute anterior circulation ischaemic stroke, established by imaging (CT scan or MRI) obtained at any time during hospitalization (reviewed by a senior neuroradiologist), with less than 7 days of clinical evolution

2. National Institutes of Health Stroke Scale (NIHSS) score [12] $\geq 4$ upon admission to the emergency department

3. Signed informed consent by the patients or their nextof-kin.

The subsequent exclusion criteria were used:

1. Previous stroke with modified Rankin scale score (mRS) [13-15] $>1$ at the time of acute stroke.

2. Brain imaging study (CT scan or MRI) with one of the following: contusion; subdural/epidural hematoma; subarachnoid haemorrhage; neoplastic lesion; infectious/ inflammatory lesion; hydrocephalus.

3. Previous history of head trauma with hospital admission.

4. Previous neurosurgery.

5. Previous history of epilepsy or epileptic seizures.

\section{Standardized clinical and ancillary evaluation}

All patients were attended by a neurologist at the emergency department and admitted at the Stroke Unit with continuous surveillance of their neurological status and daily observation by a stroke neurologist. During hospitalization, the patients underwent diagnostic tests allowing stroke etiological classification [16] and appropriate therapeutic approach, including blood tests, carotid and vertebral duplex scans, transcranial Doppler and ECG. All patients underwent a CT scan at the emergency department (1st CT scan) which was repeated $24 \mathrm{~h}$ after stroke in patients submitted to intravenous thrombolysis with alteplase (rtPA) and when clinically indicated in all patients (2nd CT scan). In selected cases, patients also performed MRI with diffusion weighted imaging, transthoracic or transesophageal echocardiography, $24 \mathrm{~h}$ Holter or cerebral angiography. NIHSS score at hospital admission, after rtPA perfusion, daily and at discharge was registered prospectively (CB and HM). During hospitalization, the following clinical, laboratory and treatment variables were daily recorded: fever/infection (respiratory, urinary, other)/organ failure (kidney, liver, heart)/withdrawal syndrome/hydroelectrolytic imbalance/hypoxemia/seizure occurrence/other medical or neurological complication/ pharmacological therapy (CB and HM).

After discharge the patient maintained standard clinical follow-up at the cerebrovascular outpatient clinic. A neurologist with expertise in epilepsy (CB) performed a telephone interview 6 months after stroke accessing seizure occurrence by a free interview followed by a brief phone screening tool for identifying patients with epilepsy [17]. A scheduled appointment 12 months after stroke was also conducted $(\mathrm{CB})$, recording the following clinical variables: NIHSS and mRS scores, occurrence of seizures and its type; other stroke or medical complications; final etiological classification of stroke [16] and on-going therapy.

\section{Neurophysiological evaluation}

All patients underwent a neurophysiological evaluation protocol that included a 64 channels video-EEG with a maximum duration of $60 \mathrm{~min}$ in different time frames after stroke:

1. As early as possible, in the first $72 \mathrm{~h}$ after admission (1st EEG).

2. Daily, after the 1st EEG, for the first 7 days after stroke (except on weekend).

3. If neurological worsening unexplained by medical complications and with indication for repeating the imaging exam.

EEGs referred in (2) and (3), were called serial EEG study during hospitalization.

4. At time of clinical discharge (discharge EEG). 
5. At 12 months after stroke (12 M EEG).

The EEG record followed national and international recommendations [18-22]. Video-EEG was performed using a Nihon-Kohden device (Neurofax EEG-1200) with a sampling frequency of $1000 \mathrm{~Hz}$. We used international 10/10 electrodes placement system and recorded at least 64 EEG channels. The total recording period was at least $35 \mathrm{~min}$ of wakefulness, including activation tests. Sleep was recorded whenever possible at the end of the exam. All records were performed by neurophysiology technicians with expertise in video-EEG and EEG records in acute brain lesion patients. Further technical specifications and EEG protocol can be read in supplementary file 1 .

\section{Imaging interpretation}

All imaging exams performed during the study period were reviewed by two seniors neuroradiologists (CC and $\mathrm{CM}$ ), blinded for clinical and electroencephalographic findings and trained for ASPECTS classification [23]. Doubts were discussed by consensus.

In patients with an infarct limited to the middle cerebral artery (MCA) territory in the imaging study (considering 1st CT scan, 2nd CT scan or MRI), the infarct size was quantified by ASPECTS [24] in 1st and 2nd CT scan. Insula and M1 to M6 ASPECTS territories were considered "cortical territories of ASPECTS". Furthermore, any type of haemorrhage transformation [25], cortical or subcortical infarct location, presence of cortical areas with normal attenuation coefficient (islands of preserved cortex) within the infarct [26-28] were evaluated in 2nd CT.

\section{Operational definitions}

1. Epileptic seizures and status epilepticus (only the first event was considered), were defined as:

1.1. Epileptic seizure; Clinical [1] and/or electrographic seizure $[29,30]$

1.2. Acute symptomatic seizure; Seizure occurring within the first 7 days of a stroke [31]. In these patients, cutoff values for metabolic disorders and febrile symptomatic seizures were not overreached and alcohol/drug withdrawal or intoxication [31] were excluded

1.3. Remote symptomatic seizure; Seizures occurring after 7 days of a stroke in the absence of precipitating factors [32]

1.4. Epilepsy; Occurrence of one unprovoked seizure and a probability of further seizures similar to the general recurrence risk (at least 60\%) after two unprovoked seizures, occurring over the next
10 years [1]. The occurrence of at least one remote symptomatic seizure or unprovoked seizure after stroke meets these criteria [2]

1.5. Status epilepticus; ILAE classification [33] and Salzburg Consensus Criteria for Non-Convulsive Status Epilepticus (NCSE) [30, 34]

2. Electroencephalographic abnormalities:

\subsection{Occurrence in the 1st EEG of:}

- Interictal epileptiforme activity (IEA) [35]; EEG transients distinguishable from background activity with a characteristic spiky morphology, namely sharp waves (duration of 70-200 ms) and spikes (duration of 20-70 ms).

- Periodic discharges (PD) [29]; Repetition of a waveform (with no more than three phases or any waveform lasting $\leq 0.5 \mathrm{~s}$ regardless of number of phases) with relatively uniform morphology and duration with a quantifiable inter-discharge interval between consecutive waveforms and recurrence of the waveform at nearly regular intervals.

- Electrographic (EEG) seizures [29, 30]; Generalized spike-wave discharges at $3 / \mathrm{s}$ or faster or clearly evolving discharges of any type that reach a frequency $>4 / s$, whether focal or generalized. Evolving was defined as at least two unequivocal, sequential changes in frequency, morphology or location lasting for at least three cycles each. Evolution in frequency was defined as at least two consecutive changes in the same direction by at least $0.5 / \mathrm{s}$. Evolution in morphology was defined as at least two consecutive changes to a novel morphology. Evolution in location was defined as sequentially spreading into or sequentially out of at least two different standard 10-20 electrode locations.

2.2. Any EEG during hospitalization (1st EEG or serial EEG study during hospitalization) with IEA and/or EEG seizures

\section{Statistical analysis}

A descriptive analysis was used for nominal qualitative and quantitative (discrete and continuous) variables. Nominal variables are expressed in frequency, the discrete variables as medians and interquartile ranges (IQR) and continuous variables as means and standard deviations (SD).

Statistical analysis was done using SPSS program version 24 for Mac. 


\section{Results}

151 patients (112 men and 39 women) with an acute anterior circulation ischaemic stroke and a mean age of 67.4 (SD 11.9) years were included. Study flowchart was previously described [36].
Demographic, clinical and imaging characteristics of these patients are displayed in Table 1.

All patients performed at least one EEG during hospitalization. The 1st EEG was performed in a median time of 1 day (IQR 1). The median number of tests performed per patient was 5 (IQR 3).
Table 1 Demographic, clinical and imaging characteristics of anterior circulation stroke patients

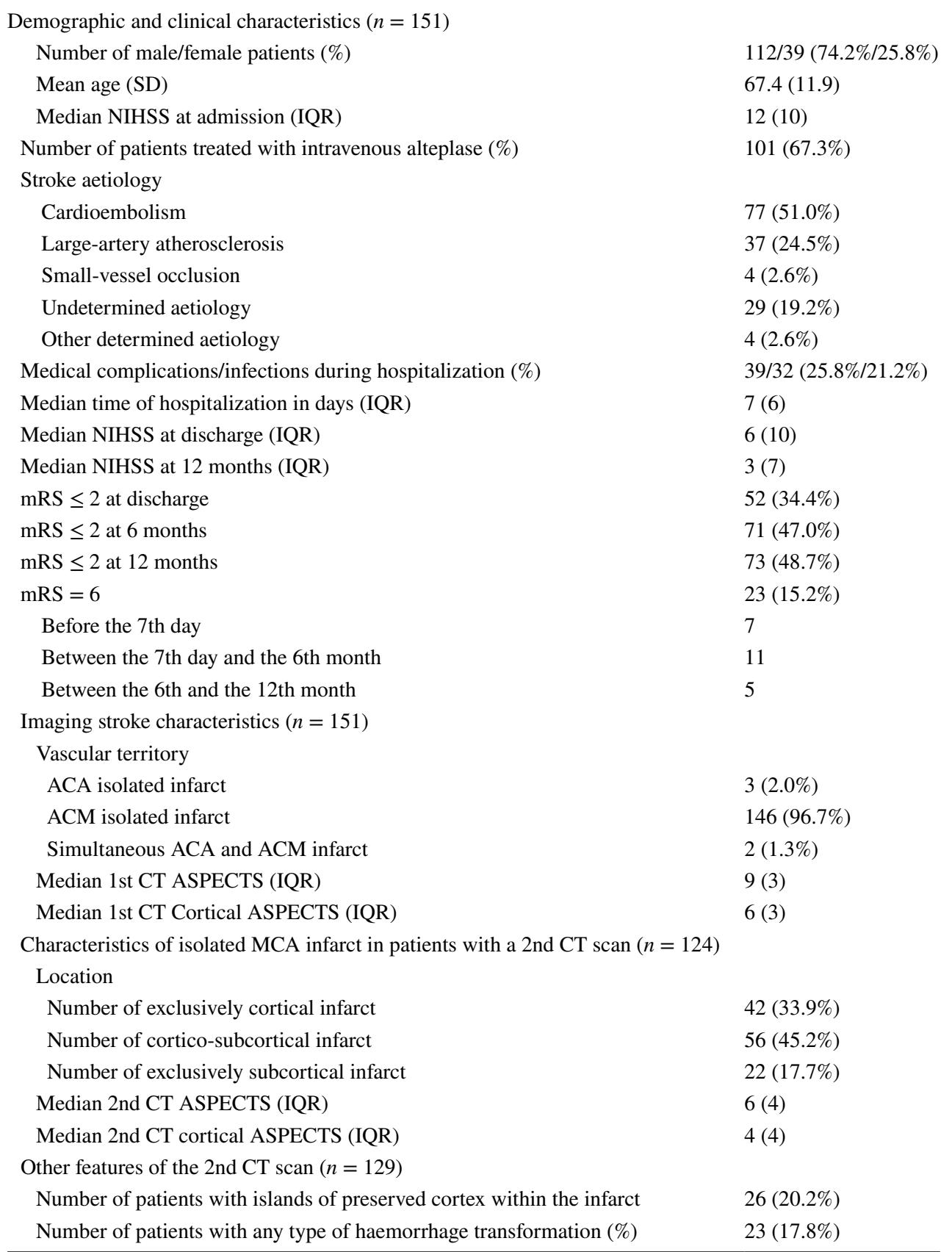

$S D$ standard deviation, NIHSS National Institutes of Health Stroke Scale score, IQR interquartile range, $m R S$ modified Rankin Scale, $A C A$ anterior cerebral artery, $M C A$ middle cerebral artery, $1 s t C T$ 1st CT scan obtain at the emergency department, ASPECTS Alberta Stroke Program Early CT Score, Cortical ASPECTS score in ASPECTS considering only the seven cortical territories of this scale, 2nd CT CT scan obtain $\geq 24 \mathrm{~h}$ after the infarct 
Of the 144 discharged patients, 143 patients $(99.3 \%)$ underwent an EEG on this date. One patient $(0.7 \%)$ refused the exam. The discharge EEG was made on average 11.1 (10.9) days after stroke (median 7).

Of the 127 patients who were alive at 12 months, 117 (92.1\%) performed an EEG at this time and 10 patients (7.9\%) refused to repeat the exam. One patient $(0.66 \%)$ was lost for clinical and neurophysiological follow-up between month 6 and 12 .

\section{Epileptic manifestations frequency}

In this study, 27 patients (17.9\%) had EEG epileptiform activity (interictal epileptiform activity and/or EEG seizures) during hospitalization. Table 2 shows the frequency of studied electroencephalographic abnormalities. Daily repetition of the EEG up to the 7th day after stroke allowed the identification of six more patients with EEG seizures not shown in the 1st EEG. Clinical and imagiological characteristics of patients with EEG seizures during hospitalization are displayed in supplementary file 2. NCSE was diagnosed in three out of the seven patients with EEG seizures (42.8\%) and to these patients anti-epileptic drugs were prescribed. The first EEG seizure occurred until the 3rd day after stroke in $85.7 \%$ of patients (in five patients on the 2 nd day and in two patients on the 3rd and 6th day after stroke, respectively).

The frequency of clinical and electroencephalographic epileptic manifestations is displayed in Table 3. One year after stroke, $23(15.2 \%)$ patients with an acute anterior ischaemic stroke had epilepsy diagnosis criteria. Seven of these epilepsy patients (30.4\%) had had an acute symptomatic seizure in the first 7 days after stroke, 2 of which
(28.6\%) were exclusively EEG seizures. In addition, 31.8\% of patients with acute symptomatic seizures ( 7 out of 22 ) also had a remote symptomatic seizure and consequently an epilepsy diagnosis.

In the first week after stroke, four patients $(2.6 \%)$ had criteria for the diagnosis of NCSE [30, 33]. In two of them, this diagnosis was made on the 1st EEG performed on the 2nd day after stroke and on other two during the first week EEG serial study (on the 3rd and the 6th day after stroke). Of the patients who met criteria for this diagnosis at the time of the 1st EEG, one had a fluctuating aphasia and periodic discharges at $3.5 \mathrm{~Hz}$ (aphasic focal NCSE) and another had consciousness impairment on the 2nd day after stroke, neither explained by imaging nor by medical complications and electrographic seizures, condition which reverted with antiepileptic treatment (focal NCSE with impaired consciousness). Of the two other patients that were in NCSE in the first week after stroke, 1 had repeated sensitive focal seizures, an EEG with periodic discharges at $2 \mathrm{~Hz}$ and electrical seizures with clinical and neurophysiological recovery after levetiracetam (focal NCSE without changing the state of consciousness). The last patient who was in NCSE during hospitalization had a malignant infarction with consciousness impairment (coma Glasgow scale score $=4$ ) and multiple seizures in the electroencephalographic recording (NCSE with coma).

\section{Discussion}

In this work, $18 \%$ of anterior circulation ischaemic stroke patients had interictal or ictal epileptiform activity in the EEG during hospitalization and $25 \%$ at least one seizure in

Table 2 EEG abnormalities in different time frames after stroke

\begin{tabular}{|c|c|c|c|c|c|c|c|}
\hline & $\begin{array}{l}\text { 1st EEG } \\
n(\%)\end{array}$ & $\begin{array}{l}\text { Serial EEG study dur- } \\
\text { ing hospitalization } \\
n(\%)\end{array}$ & $\begin{array}{l}\text { Mc. Nemar's } \\
\text { test } 1 \\
p\end{array}$ & $\begin{array}{l}\text { Discharge EEG } \\
n(\%)\end{array}$ & $\begin{array}{l}\text { Mc. Nemar's } \\
\text { test } 2 \\
p\end{array}$ & $\begin{array}{l}12 \mathrm{M} \text { EEG } \\
n(\%)\end{array}$ & $\begin{array}{l}\text { Mc. } \\
\text { Nemar's } \\
\text { test } 3 \\
p\end{array}$ \\
\hline Total of patients with & $151(100)$ & $151(100)$ & - & $143(94.7)$ & - & $116(76.8)$ & - \\
\hline PD & $27(17.9)$ & $38(25.2)$ & 0.007 & $9(6.3)$ & 0.002 & $3(2.6)$ & 0.002 \\
\hline IEA & $16(10.6)$ & $18(11.9)$ & ns & $12(8.4)$ & ns & $5(4.3)$ & ns \\
\hline EEG seizures & $1(0.7)$ & $6(4.0)$ & ns & 0 & ns & 0 & ns \\
\hline NCSE criteria & $2(1.3)$ & $2(1.3)$ & ns & 0 & ns & 0 & ns \\
\hline
\end{tabular}

1st EEG video-EEG ( $<60 \mathrm{~min}$ ) performed as early as possible, in the first $72 \mathrm{~h}$ after admission for acute anterior circulation ischaemic stroke, Serial EEG during hospitalization video-EEG ( $<60 \mathrm{~min})$ performed daily for the first 7 days after stroke (except on weekend) or if neurological worsening unexplained by medical complications and with indication for repeating the imaging exam (at least one EEG record during the hospitalization with one of the analysed features), Mc. Nemar's test 1 Mc. Nemar's test defining the difference between 1 st EEG and serial EEG during hospitalization, Discharge EEG video-EEG ( $<60 \mathrm{~min})$ performed at time of clinical discharge, Mc. Nemar's test 2 Mc. Nemar's test defining the difference between 1st EEG and discharge EEG, 12 M EEG video-EEG ( $<60 \mathrm{~min})$ performed at 12 months after stroke, Mc. Nemar's test $3 \mathrm{Mc}$. Nemar's test defining the difference between 1st EEG and 12 months EEG, $P D$ periodic discharges, IEA interictal epileptiforme activity, EEG Seizures electrographic seizures, NCSE non-convulsive status epilepticus. Four patients $(2.6 \%)$ had NCSE criteria during hospitalization. Of these, three had EEG seizures and one patient periodic discharges $\geq 3 \mathrm{~Hz}$ in the 1st EEG, $n s$ non-significant $(p>0.05)$ 
Table 3 Frequency of clinical and EEG epileptic manifestations in anterior circulation stroke patients

\begin{tabular}{ll}
\hline Type of epileptic manifestation & $n(\%)$ \\
\hline At least one epileptic seizure in the first year after stroke & $38(25.2 \%)$ \\
& $33(86.8 \%)$ exclusively clinical seizures \\
Acute symptomatic seizure (at least one) & $5(13.2 \%)$ exclusively EEG seizures \\
& $22(14.6 \%)$ \\
& $17(77.3 \%)$ exclusively clinical seizures \\
Remote symptomatic seizure as the first seizure & $5(22.7 \%)$ exclusively EEG seizures \\
Remote symptomatic seizure (at least one remote symptomatic & $13(59.1 \%)$ occurred in the first $24 \mathrm{~h}$ \\
seizure, with or without a previous acute symptomatic seizure) & $23(15.2 \%)$ \\
& 7 Also acute symptomatic seizures (5 \\
& $11(47.8 \%)$ between day 7 and 6 th \\
IEA in the 1st EEG & month \\
EEG seizure within the first 7 days of stroke & $12(52.2 \%)$ between the 6th and 12th \\
IEA or EEG seizure during hospitalization & month \\
\hline
\end{tabular}

EEG seizures electrographic seizures, IEA interictal epileptiform activity the first year after stroke. Furthermore, more than $20 \%$ of acute symptomatic seizures were exclusively electrographic and more than $40 \%$ of patients with EEG seizures had NCSE criteria or remote symptomatic seizures. Our results support the hypothesis that in the absence of a neurophysiological evaluation, the frequency of acute symptomatic seizures after stroke is underestimated.

Several strengths are identified in this work including the sample size of anterior circulation acute stroke patients, with prospective clinical and EEG follow-up, unlike previous studies (supplementary file 3 and 4 ), and the small number of patients lost for clinical follow-up $(n=1)$. Another aspect that stands out is the use of internationally recognized terminology for EEG description [29]. This terminology not only shows a good inter-observer agreement $[37,38]$ as it is recommended for multicentric research on EEG patterns in acute neurological disease patients [37] and for implementation in clinical practice [38]. In addition, the time period for classification of seizures as acute or remote symptomatic is in accordance with the ILAE recommendations [31] and only acute anterior circulation infarcts established by imaging were included.

There are some limitations in this study. The serial and non-continuous nature of the neurophysiological assessment may in fact be considered a constraint. However, using a single EEG with less than 60 min duration we found the same percentage of patients with periodic discharges and epileptiform activity as Carrera et al. [39] in patients undergoing continued EEG monitoring for over $17 \mathrm{~h}$. Thus, our work suggests that a briefer EEG, performed in a short time window after stroke, can provide similar information than a longer record, with the advantage of being technically easier and less expensive. Nevertheless, periodic discharges and epileptiform activity have different specificities in seizure prediction. Although periodic discharges have been described in the continuum between an interictal and ictal phenomenon [40], this activity may be an acute cerebral lesion signature [41]. For this reason we clearly defined and distinguished interictal epileptiform activity and periodic discharges. Nevertheless, the percentage of interictal epileptiforme activity found in our study is not to much different from Carrera et al. [39] (10.6 vs. 14\%). Future studies should compare the performance of short duration (spot) EEG versus a continuous one in the detection of epileptic manifestations.

In our series, $22.7 \%$ of acute symptomatic seizures were exclusively electroencephalographic and occurred for the first time in the majority $(85.7 \%)$ of patient in the first $72 \mathrm{~h}$ after stroke. An intensive care unit study, with continuous EEG also showed that $89 \%$ of seizures occurred within $72 \mathrm{~h}$ [42]. These observations show the importance of a neurophysiological evaluation, particularly in the first 3 days after stroke. Furthermore, in our study, almost $1 / 3$ $(31.8 \%)$ of patients having a seizure in the first seven days after stroke had an epilepsy diagnosis 1 year after stroke, in accordance to Hesdorffer et al. [2] which found a 33\% risk of an unprovoked seizure in patients with a first post-stroke acute symptomatic seizure. Additionally, our results showed that more than $1 / 4(28,6 \%)$ of post-stroke acute symptomatic seizure patients that had a vascular epilepsy diagnosis in 1 year time period, would not have been identified without the EEG protocol that was used.

However, in our study, the frequency of EEG seizures $(4.6 \%)$ is lower than that reported in continuous EEG 
studies in ischaemic stroke ranging between 6 to $27 \%$ [7, 39, 42-46] (supplementary file 3 ). This observation was expected since, comparatively with 1 st short duration EEG, a continuous record detected twice more seizures in a population of intensive care unit patients [44]. However, it is possible that the low number of patients with EEG seizures is not exclusively due to a shorter EEG duration but also to our study setting (a neurology department stroke unit) and to the inclusion of less severe stroke patients than intensive care units. Furthermore, different definitions of ictal epileptic activity can also account for the lower amount of detected EEG seizures in our study. The evidence favouring continuous versus spot EEG in detecting seizures is limited [47], especially in patients with ischemic stroke admitted to non intensive profile services as it was the case of our patients. Still, continuous EEG it is not accessible at all centres and its cost-benefit is not determined [48].

In our study, $42.9 \%$ of patients with EEG seizures fulfilled criteria for the diagnosis of NCSE or had remote symptomatic seizures in the clinical follow-up. In fact, non-convulsive status epilepticus has been described as one of the major diagnostic and therapeutic challenges in modern neurology [49] and the EEG is essential for its diagnosis. The described association between post-stroke status epilepticus and functional prognosis [50-52], reinforces the importance of early recognition of this entity, allowing appropriate and timely treatment. Due to very low clinical evidence, current ESO guidelines [3] only give weak recommendations on secondary prevention of acute symptomatic post-stroke seizures. Their treatment is frequently decided on an individual basis guided but the presence of intermittent or persistent altered mental status and fluctuating recovery or status epilepticus diagnosis [4]. In our study, more than $40 \%$ of patients with EEG seizures had NCSE criteria and $75 \%$ of patients with NCSE (three out four) had no obvious clinically acute poststroke symptomatic seizures, showing the usefulness of our eletrophysiological study.

Acknowledgements We are obliged to EEG technicians Joana Pires, Lígia Ferreira e Rosa Santos. We are also grateful to all nurses and medical residents working in the Stroke Unit between 2011 and 2013 for their support to this Project.

\section{Compliance with ethical standards}

Funding The 2012 Research Grant in Cerebrovascular Diseases (scientific promoter: Stroke Portuguese Society (SPAVC)/Sponsor: Tecnifar), supported this work.

Conflicts of interest Dr. Bentes received the 2012 Research Grant in Cerebrovascular Diseases (Scientific Promoter: Sociedade Portuguesa do AVC/Sponsor: Tecnifar). Dr. Ferro reports personal fees from Boehringer Ingelheim, outside the submitted work. Other authors have nothing to disclose.
Ethics approval This study has been approved by the Ethics Committee "Comissão de Ética para a Saúde" of the HSM-CHLN and has, therefore, been preformed in accordance with the ethical standards laid down in the 1964 Declaration of Helsinki and its later amendments.

\section{References}

1. Fisher RS, Acevedo C, Arzimanoglou A et al (2014) ILAE Official Report: a practical clinical definition of epilepsy. Epilepsia 55:475-482

2. Hesdorffer DC, Benn EKT, Cascino GD, Hauser WA (2009) Is a first acute symptomatic seizure epilepsy? Mortality and risk for recurrent seizure. Epilepsia 50:1102-1108

3. Holtkamp M, Beghi E, Benninger F et al (2017) European Stroke Organisation guidelines for the management of post-stroke seizures and epilepsy. Eur Stroke J 2:103-115

4. Chung JM (2014) Seizures in the acute stroke setting. Neurol Res 36:403-406

5. Camilo O, Goldstein LB (2004) Seizures and epilepsy after ischemic stroke. Stroke 35:1769-1775

6. Pitkänen A, Roivainen R, Lukasiuk K (2015) Development of epilepsy after ischaemic stroke. Lancet Neurol 15:185-197

7. Claassen J, Mayer S, Kowalski RG et al (2004) Detection of electrographic seizures with continuous EEG monitoring in critically ill patients. Neurology 62:1743-1748

8. Huang CW, Saposnik G, Fang J et al (2014) Influence of seizures on stroke outcomes: a large multicenter study. Neurology 82:768-776

9. Young GB, Jordan KG, Doig GS (1996) An assessment of nonconvulsive seizures in the intensive care unit using continuous EEG monitoring: an investigation of variables associated with mortality. Neurology 47:83-89

10. Oddo M, Carrera E, Claassen J et al (2009) Continuous electroencephalography in the medical intensive care unit. Crit Care Med 37:2051-2056

11. Kamel H, Betjemann JP, Navi BB et al (2013) Diagnostic yield of electroencephalography in the medical and surgical intensive care unit. Neurocrit Care 19:336-341

12. Goldstein L, Bertels C, Davis J (1989) Interrater reliability of the NIH stroke scale. Arch Neurol 46:660-662

13. Banks JL, Marotta CA (2007) Outcomes validity and reliability of the modified rankin scale: implications for stroke clinical trials-a literature review and synthesis. Stroke 38:1091-1096

14. Rankin J (1957) Cerebral vascular accidents in patients over the age of 60: II. Prognosis. Scottish Med J 2:200-215

15. van Swieten JC, Koudstaal PJ, Visser MC et al (1988) Interobserver agreement for the assessment of handicap in stroke patients. Stroke 19:604-607

16. Adams H, Bendixen B, Kappelle L et al (1993) Classification of subtype of acute ischemic stroke. Stroke 23:35-41

17. Ottman R, Barker-Cummings C, Leibson CL et al (2010) Validation of a brief screening instrument for the ascertainment of epilepsy. Epilepsia 51:191-197

18. Nuwer M, Comi G, Emerson R et al (1998) IFCN standards for digital recording of clinical EEG. International Federation of Clinical Neurophysiology. Electroencephalogr Clin Neurophysiol 106:259-261

19. Flink R, Pedersen B, Guekht B et al (2002) Guidelines for the use of EEG methodology in the diagnosis of epilepsy. International League Against Epilepsy: commission report. Commission on European Affairs: subcommission on European Guidelines. Acta Neurol Scand 106:1-7 
20. American Clinical Neurophysiology Society (2006) Guideline 4: standards of Practice in Electroencephalography. http://www.acns. org/pdf/guidelines/Guideline-4.pdf. Accessed 05 Aug 2017

21. American Clinical Neurophysiology Society (2006) Guideline 5: guidelines for standard electrode position nomenclature. Clin Neurophysiol 6:1-3

22. Martins da Silva A, Bentes C, Atalaia A, Mendes-Ribeiro JA (2011) Recomendações para utilização do electroencefalograma (EEG) em Epilepsia: Relatório à Liga Portuguesa Contra a Epilepsia. http://static.lvengine.net/epilepsia/Imgs/pages/page_105/ comisso-de-eeg_lpce_v1.pdf. Accessed 05 Aug 2017

23. Alberta Health Services (2016) University of calgary medicine faculty training for aspects. http://www.aspectsinstroke.com/ training-for-aspect. Accessed 10 Apr 2016

24. Barber P, Demchuk A, Zhang J, Buchan A (2000) Validity and reliability of a quantitative computed tomography score in predicting outcome of hyperacute stroke before thrombolytic therapy. ASPECTS Study Group. Alberta Stroke Programme Early CT Score. Lancet 355:1670-1674

25. Anon (2002) The SITS Monitoring Study (SITS-MOST) Final protocol. http://www.acutestroke.org/SM_Protocol/SITS-MOST_ final_protocol.pdf. Accessed 05 Aug 2017

26. Ryglewicz D, Baranska-Gieruszczak M, Niedzielska K, KrystWidzgowska T (2013) EEG and CT findings in poststroke epilepsy. Acta Neurol Scand 81:488-490

27. Awada A, Omojola MF, Obeid T (1999) Late epileptic seizures after cerebral infarction. Acta Neurol Scand 99:265-268

28. Pohlmann-Eden B, Fatar M, Hennerici M (2001) The preserved cortical island sign is highly predictive of postischemic seizures. Cerebrovasc Dis 12:282

29. Hirsch LJ, LaRoche SM, Gaspard N et al (2013) American Clinical Neurophysiology Society's Standardized Critical Care EEG Terminology: 2012 version. J Clin Neurophysiol 30:1-27

30. Beniczky S, Hirsch LJ, Kaplan PW et al (2013) Unified EEG terminology and criteria for nonconvulsive status epilepticus. Epilepsia 54:28-29

31. Beghi E, Carpio A, Forsgren L et al (2010) Recommendation for a definition of acute symptomatic seizure. Epilepsia 51:671-675

32. Hauser WA, Beghi E (2008) First seizure definitions and worldwide incidence and mortality. Epilepsia 49:8-12

33. Trinka E, Cock H, Hesdorffer D et al (2015) A definition and classification of status epilepticus-Report of the ILAE Task Force on Classification of Status Epilepticus. Epilepsia 56:1515-1523

34. Leitinger M, Beniczky S, Rohracher A et al (2015) Salzburg consensus criteria for non-convulsive status epilepticus-approach to clinical application. Epilepsy Behav 49:158-163

35. Noachtar S, Binnie C, Ebersole J et al (1999) A glossary of terms most commonly used by clinical electroencephalographers and proposal for the report form for the EEG findings. The International Federation of Clinical Neurophysiology. Electroencephalogr Clin Neurophysiol Suppl 52:21-41
36. Bentes C, Martins H, Peralta AR et al (2017) Epileptic manifestations in stroke patients treated with intravenous alteplase. Eur J Neurol 24(6):755-761

37. Gaspard N, Hirsch LJ, LaRoche SM et al (2014) Interrater agreement for critical care EEG terminology. Epilepsia 55:1366-1373

38. Leitinger M, Trinka E, Gardella E et al (2016) Diagnostic accuracy of the Salzburg EEG criteria for non-convulsive status epilepticus: a retrospective study. Lancet Neurol 15:1054-1062

39. Carrera E, Michel P, Despland P et al (2006) Continuous assessment of electrical epileptic activity in acute stroke. Neurology 67:99-104

40. Chong D, Hirsch L (2005) Which EEG patterns warrant treatment in the critically ill? Reviewing the evidence for treatment of periodic epileptiform discharges and related patterns. J Clin Neurophysiol 22:79-91

41. Hartings JA, Williams AJ, Tortella FC (2003) Occurrence of nonconvulsive seizures, periodic epileptiform discharges, and intermittent rhythmic delta activity in rat focal ischemia. Exp Neurol 179:139-149

42. Vespa PM, O'Phelan K, Shah M et al (2003) Acute seizures after intracerebral hemorrhage: a factor in progressive midline shift and outcome. Neurology 60:1441-1446

43. Kurtz P, Gaspard N, Wahl AS et al (2014) Continuous electroencephalography in a surgical intensive care unit. Intensive Care Med 40:228-234

44. Pandian JD, Cascino GD, So EL et al (2004) Digital video-electroencephalographic monitoring in the neurological-neurosurgical intensive care unit. Arch Neurol 61:1090-1094

45. Swisher CB, Shah D, Sinha SR, Husain AM (2015) Baseline EEG pattern on continuous ICU EEG monitoring and incidence of seizures. J Clin Neurophysiol 32:147-151

46. Westover MB, Shafi MM, Bianchi MT et al (2015) The probability of seizures during EEG monitoring in critically ill adults. Clin Neurophysiol 126:463-471

47. Claassen J, Vespa P (2014) Electrophysiologic monitoring in acute brain injury. Neurocrit Care 21:129-147

48. Grillo E (2015) Concerns about utility and cost-effectiveness of continuous critical-care EEG. J Clin Neurophysiol 32:442-443

49. Meierkord H, Holtkamp M (2007) Non-convulsive status epilepticus in adults: clinical forms and treatment. Lancet Neurol 6:329-339

50. Velioglu SK, Ozmenoglu M, Boz C, Alioglu Z (2001) Status epilepticus after stroke. Stroke 32:1169-1172

51. Rumbach L, Sablot D, Berger E et al (2000) Status epilepticus in stroke: report on a hospital-based stroke cohort. Neurology 54:350-354

52. Knake S, Rochon J, Fleischer S et al (2006) Status epilepticus after stroke is associated with increased long-term case fatality. Epilepsia 47:2020-2026 